hep-th/9710005

IASSNS-HEP-97/103

\title{
The Matrix Formulation of Type IIB Five-Branes
}

\author{
Savdeep Sethi* \\ School of Natural Sciences \\ Institute for Advanced Study \\ Princeton, NJ 08540, USA
}

\begin{abstract}
We present a matrix model which interpolates between type IIA and type IIB NS fivebranes. The matrix description involves a three-dimensional bulk quantum field theory interacting with impurities localized in one spatial direction. We obtain a dual matrix formulation for the exotic six-dimensional theory on coincident type IIB NS five-branes by studying the T-dual description in terms of Kaluza-Klein monopoles in type IIA string theory. After decoupling the bulk physics, the matrix description reduces to the conformal field theory of the Coulomb branch for the type IIA matrix string propagating on certain singular spaces. In many ways, this dual realization of superconformal theories is the two-dimensional analogue of three-dimensional mirror symmetry.
\end{abstract}

$9 / 97$

* sethi@sns.ias.edu 


\section{Introduction}

Exotic six-dimensional theories have received increased attention recently. The most studied of these theories is the $(2,0)$ field theory, which was first discovered in [1,2]. This field theory is an interacting superconformal theory living on $k$ coincident five-branes in $\mathrm{M}$ theory. In large part, the reason this theory is so interesting is that it remains interacting in the limit where the eleven-dimensional Planck constant, $M_{p l}$, is taken to infinity. In this limit, the theory living on the five-branes decouples from the bulk spacetime modes, leaving a consistent, complete theory in six-dimensions. This type of decoupling argument has been applied to the five-branes in string theory to argue for the existence of new six-dimensional theories [3]. The theory on $k$ parallel branes is associated to an $A_{k-1}$ singularity, but similar six-dimensional theories can be associated to $D$ and $E$ singularities. Aspects of the $(2,0)$ field theory associated to $D$ and $E$ singularities have been studied in 《1, 迎.

The aim of this paper is to consider the case of six-dimensional theories with $(1,1)$ supersymmetry. As before, the theories associated to $A_{k-1}$ singularities can be realized in terms of coincident NS five-branes in type IIB string theory. The existence of complete theories on type IIB NS five-branes has been argued in [3]. The fields in a theory with $(1,1)$ supersymmetry in six-dimensions and no gravity must appear in vector supermultiplets. At low-energies, the theory on type IIB five-branes is therefore a gauge theory. The gauge coupling for the $U(k)$ gauge theory living on $k$ parallel branes depends only on the string scale, $M_{s}$ [3]. Since the gauge coupling is independent of the type IIB string coupling, $g_{s}^{B}$, the theory on the branes remains interacting in the limit where the string coupling vanishes and $M_{p l} \rightarrow \infty$. This is to be constrasted with the theories of tensor multiplets, where the self-duality constraint on the three-form field strength guarantees that these theories remain interacting when $M_{p l} \rightarrow \infty$. In addition to the vector particles, the sixdimensional theory includes strings of tension $M_{s}^{2}$, which can be viewed as bound states of fundamental strings with the NS five-branes, or alternately, as instantons of the $U(k)$ gauge theory. Our goal is to find a matrix description of five-branes in type IIB string theory. From this matrix description, we can extract a matrix definition for the exotic $(1,1)$ theory associated to coincident NS five-branes. We will extend the definition to the theories associated to general $A-D-E$ singularities. The decoupling limit for matrix theories involving five-branes in $\mathrm{M}$ theory, type IIA and heterotic string theory has been discussed recently in [5-9], partly motivated by earlier work [10]. 
In obtaining a matrix description for these interacting six-dimensional theories, we will find dual realizations of certain superconformal field theories with eight supersymmetries in two dimensions. One realization is a limit of a $2+1$-dimensional theory with impurities localized in one space dimension. The bulk theory has sixteen supersymmetries, but the impurities break half the supersymmetries. The second realization is in terms of a $1+1$-dimensional theory of vector and hypermultiplets with $(4,4)$ supersymmetry. In many ways, this dual description of certain superconformal fixed points is the two-dimensional analogue of the three-dimensional mirror symmetry found by Intriligator and Seiberg [11]. Observations related to those presented here have been made by Witten [12]. As I completed this project, related work appeared in [13].

\section{Matrix Models for Type IIB Five-Branes}

\subsection{The $M$ theory construction of type IIB five-branes}

Let us begin by considering the $\mathrm{M}$ theory construction of five-branes in type IIB string theory. $\mathrm{M}$ theory compactified on a two-torus is equivalent to the type IIB string on a circle whose size grows inversely with the volume of the two-torus [14]. The torus, $T^{2}$, on which we are compactifying is defined by its complex structure, $\tau$, and its volume. For simplicity, let us take the torus to be rectangular with sides of length, $2 \pi R_{1}$ and $2 \pi R_{2}$. Since we will eventually restrict to NS five-branes, this choice of complex structure will not affect the following discussion in a significant way. The radius of the type IIB circle is,

$$
R_{B}=\frac{1}{M_{p l}^{3} R_{1} R_{2}}=\frac{1}{M_{s}^{2} R_{2}} .
$$

The string scale is defined in terms of $M_{p l}$ and $R_{1}$ by the relation:

$$
M_{s}^{2}=R_{1} M_{p l}^{3} \text {. }
$$

The type IIB string coupling is given by, $g_{s}^{B}=R_{1} / R_{2}$. There are now two ways to realize a $(p, q)$ five-brane in the type IIB theory. The first realization is in terms of an $\mathrm{M}$ theory five-brane wrapped on a $(p, q)$ cycle of the two-torus. This corresponds to a $(p, q)$ fivebrane in type IIB string theory wrapped on the circle with radius $R_{B}$. Note that in these conventions, an M theory five-brane wrapped on $R_{2}$ results in a type IIB NS five-brane. When the area of the torus is taken to zero with $M_{s}$ held fixed, we obtain a $(p, q)$ five-brane in flat non-compact space. 
The second realization is obtained by wrapping an $\mathrm{M}$ theory six-brane along a $(p, q)$ cycle of the torus. This corresponds to a $(p, q)$ five-brane in the type IIB theory with a compact transverse dimension of radius $R_{B}$. Again, taking the area of the torus to zero results in a $(p, q)$ five-brane in flat non-compact spacetime. We will examine the matrix realization for this second case in a limit that captures the decoupled physics in the following section. Since both ways of constructing a $(p, q)$ five-brane must be equivalent, the corresponding matrix descriptions of the decoupled theory must also be equivalent. This equivalence gives a two-dimensional analogue of the three-dimensional mirror symmetry found by Intriligator and Seiberg [11].

The limit in which the bulk physics decouples from the six-dimensional theory on the five-branes has been described in [3]. We need to take,

$$
\begin{aligned}
& M_{p l} \rightarrow \infty, \\
& g_{s}^{B} \rightarrow 0,
\end{aligned}
$$

while holding fixed $M_{s}$. We should then find a family of new theories parametrized by $M_{s}$. In case one, we also need to take the area of the torus to zero to obtain a five-brane with no compact internal dimensions. For case two, the size of the transverse circle is irrelevant in the decoupling limit [7]. In this case, the moduli space for the gauge theory on $k$ coincident branes might seem to be,

$$
\mathcal{M}=\frac{\left(\mathbb{R}^{3} \times S^{1}\right)^{k}}{\mathbf{S}_{k}},
$$

with compact directions. In actuality, the period for the compact scalars contains a factor of the inverse string coupling and so decompactifies in the decoupling limit. We are therefore free to take $R_{B}$ to any convenient value. We shall exploit this freedom to solve for the matrix description of the theory on NS five-branes.

\subsection{NS five-branes in type IIB matrix theory}

Let us turn to the matrix description of case one. This involves a rather interesting, slightly novel theory, which is similar in many ways to systems with impurities which are studied in condensed matter physics. Similar systems appear in matrix descriptions of Yang-Mills theories in less than six space-time dimensions, but that is a topic to be explored elsewhere [15]. The matrix theory for the type IIB string without any five-branes has been considered in [16,17], generalizing the original matrix conjecture for uncompactified 
$\mathrm{M}$ theory [18]. The parameters of the matrix theory are fixed in terms of the size of the longitudinal direction, $R$, and the two radii of the torus, $R_{1}$ and $R_{2}$. The matrix description is a $2+1$-dimensional $U(N)$ gauge theory with sixteen supersymmetries. This gauge theory will be our bulk theory. The parameter $N$ is the number of zero-branes. At least for cases with sixteen supersymmetries, the parameter $N$ need not be taken to infinity if the matrix model is viewed as describing $M$ theory quantized in the discrete light-cone formalism [19]. The gauge theory lives on a torus with radii,

$$
\Sigma_{i}=\frac{1}{M_{p l}^{3} R_{i} R},
$$

and the coupling constant for the bulk gauge theory is given by

$$
g_{Y M}^{2}=\frac{R}{R_{1} R_{2}}=R^{3} M_{p l}^{6} \Sigma_{1} \Sigma_{2}
$$

For studying the decoupling limit, it is convenient to express these parameters in string units where,

$$
\begin{aligned}
& \Sigma_{1}=\frac{1}{M_{s}^{2} R} \\
& \Sigma_{2}=\frac{1}{M_{s}^{2} R} g_{s}^{B} .
\end{aligned}
$$

The presense of parallel $\mathrm{M}$ theory five-branes breaks half of the supersymmetries in the Yang-Mills theory. The matrix theory for the case where the $k$ five-branes are placed transverse to the two-torus has been discussed in [7]. It is a generalization of the quantum mechanics for a longitudinal five-brane which describes the coupling of zero-branes to a background type IIA four-brane [20]. In this situation, the five-branes are represented by $k$ hypermultiplets in the fundamental of the gauge group. These $2+1$-dimensional hypermultiplets come from the quantum mechanical hypermultiplets representing the $0-4$ strings. The quantum mechanical degrees of freedom are promoted to fields because of the two compact transverse dimensions [21,18]. The spacetime physics is encoded in the structure of the Coulomb branch which, as a function of $R_{2}$, interpolates between the metric for the type IIB Kaluza-Klein monopole and the tube metric of the type IIA NS five-brane [22]. When there is more than one coincident brane, there is also a Higgs branch which describes the physics localized on the five-branes. It is interesting to note that these theories have been studied in the context of three-dimensional mirror symmetry [11], where candidate duals have been conjectured [23]. 
In our case, we want type IIB NS five-branes, so we consider $k$ longitudinal M theory five-branes wrapped on $R_{2}$. After T-duality on $R_{2}$, the $0-4$ strings become hypermultiplets located at points points on $\Sigma_{2}$. The $N$ zero-branes become D-strings wrapping $\Sigma_{2}$. A further T-duality on $R_{1}$ promotes the $0-4$ strings to $1+1$-dimensional hypermultiplets located at points on $\Sigma_{2}$. The $N$ zero-branes become two-branes wrapping the dual torus. From the perspective of the bulk theory, the hypermultiplets are localized impurities which break half of the supersymmetries. The position of the $k$ points on $\Sigma_{2}$ is determined by the choice of flat connection for the $U(k)$ gauge-field living on the wrapped four-brane system. Generally, when the four-branes are not coincident, some of the hypermultiplets are massive.

After this sequence of T-dualities, this theory becomes one containing $N$ two-branes wrapped on $T^{2}$ with $k$ four-branes located at points on $\Sigma_{2}$, and wrapped along the $\Sigma_{1}$ direction. When there is more than one four-brane, the two-branes can 'break' at the positions of the wrapped four-branes in a way which is essentially T-dual to the breaking of D-strings wrapped on a circle at the location of three-branes located at points on the circle. When the bulk gauge coupling is taken to infinity, this type IIA configuration of four-branes and two-branes is better described in M theory since the eleventh dimension decompactifies. This is analogous to the situation in [24], but here the result is a system of five-branes with membranes stretched between them.

For simplicity, let us place the $k$ longitudinal five-branes at the same point in the transverse space,

$$
x_{6}=\ldots=x_{9}=0 .
$$

We can place $k_{p}$ five-branes at the point $x_{2}=x_{p}$, where $x_{2}$ is a coordinate for the circle with radius $\Sigma_{2}$ and $\sum k_{p}=k$. The bulk Yang-Mills theory has seven scalars, $\phi_{i}$, in the adjoint of $U(N)$ which transform under a global $\operatorname{Spin}(7)$ symmetry. In the presence of the five-branes, the global symmetry decomposes into $\operatorname{Spin}(3) \times \operatorname{Spin}(4)$ where the $\operatorname{Spin}(3)$ rotates $\phi_{3}, \phi_{4}, \phi_{5}$, while $\operatorname{Spin}(4)$ acts on $\phi_{6}, \phi_{7}, \phi_{8}, \phi_{9}$. These latter scalars parametrize motion away from the five-branes. Since we are interested in the decoupled physics, we will freeze the expectation values for these fields at the position of the longitudinal fivebranes. Since the spatial directions are compact, the notion of a well-defined expectation value exists only classically. The Lagragian for this system of impurities interacting with the bulk modes can be obtained from the original $0-0$ and $0-4$ lowest string modes using an extension of the method described in [21, 18, 25]. We start with the positions of $\mathrm{N}$ zerobranes in $\mathbb{R}^{9}$ described by nine matrices, $X^{i}$, in the adjoint of $U(N)$. In addition, we have 
$k$ hypermultiplets $Q_{A}^{f}$ which are complex bosons in the fundamental of $U(N)$. The flavor index, $f=1, \ldots, k$ while $A=1,2$ is an index for the doublet representation of $S U(2)_{R}$. Compactifying $X^{1}$ is accomplished by taking an array of $0-4$ systems consisting of the original system and all its translates shifted along $X^{1}$ by $2 \pi n \Sigma_{1}$ for all integer $n$. The gauge group then becomes infinite-dimensional, and the $X^{i}$ for $i>1$ and hypermultiplets $Q^{f}$ can be organized into fields depending on the periodic coordinate $x_{1}$. Lastly, $X^{1}$ can be replaced by the connection, $-i \partial_{1}-A_{1}\left(x_{1}\right)$. In this way, we obtain $1+1$-dimensional Yang-Mills coupled to $k$ hypermultiplets. A similar story occurs for the $X^{i}$ fields when $X^{2}$ is compactified. The $X^{i}$ for $i>2$ become $2+1$-dimensional fields $\phi_{i}$ depending on $x_{1}, x_{2}$ while $X^{2}$ is replaced by the connection, $-i \partial_{2}-A_{2}$. These bulk fields have a standard Lagrangian whose bosonic part is,

$$
L_{\text {bulk }}=\int d t d x_{1} d x_{2}\left(-\frac{1}{4} \operatorname{Tr}\left(F_{\mu \nu}^{2}\right)-\frac{1}{2} \operatorname{Tr}\left(D_{\mu} \phi_{i}\right)^{2}-\frac{1}{2} \sum_{i<j} \operatorname{Tr}\left[\phi_{i}, \phi_{j}\right]^{2}\right) .
$$

However, the hypermultiplets now need to be treated differently. Let us take the $k_{p}$ hypermultiplets located at $x_{p}$. There are similar expressions for the remaining localized hypermultiplets. The terms in the Lagrangian that come from the reduction of the sixdimensional kinetic terms are,

$$
L_{\text {impurity }}=\int d t d x_{1}\left(-\sum_{\mu=0,1}\left|D_{\mu} Q_{A}^{f_{p}}\left(x_{1}, t\right)\right|^{2}-\sum_{i=6}^{9}\left|\phi_{i}\left(x_{1}, x_{p}, t\right) Q_{A}^{f_{p}}\right|^{2}\right),
$$

where $f_{p}=1, \ldots, k_{p}$ and where the connection is evaluated at $x_{2}=x_{p}$. There are two more terms in the potential. The first is proportional to, 1

$$
\sum_{a, A, B} Q_{A f_{p}}^{\dagger} T^{a} Q_{B}^{f_{p}} Q^{A f_{p}^{\prime}} T^{a} Q_{f_{p}^{\prime}}^{B \dagger}
$$

where the $T^{a}$ are the generators for the fundamental representation. The final term is the most interesting, and is proportional to,

$$
\sum_{l, m=1}^{4} Q^{A f_{p}}\left[\tilde{\phi}_{l}, \tilde{\phi}_{m}\right] \tau_{A B}^{l m} Q_{f_{p}}^{B \dagger}+\text { c.c. }
$$

1 The proportionality constant includes a $\delta(0)$ factor which is essentially the order of the symmetry group. This divergent factor is needed to obtain a Higgs branch with the expected physical properties [26]. 
The fields $\tilde{\phi}$ are,

$$
\begin{aligned}
& \tilde{\phi}_{1}=D_{2}\left(x_{1}, x_{p}, t\right) \\
& \tilde{\phi}_{r}=\phi_{r+1}\left(x_{1}, x_{p}, t\right) \quad r=2,3,4 .
\end{aligned}
$$

To the localized hypermultiplets, the gauge-field $A_{2}$ is a scalar filling out a hypermultiplet that parametrizes the position of the zero-branes in the longitudinal five-brane. As before, the Coulomb branch of the bulk - including the interactions with the localized hypermultiplets - should describe the spacetime physics in the background of $k$ type IIB NS five-branes wrapped on $R_{B}$. As a function of $R_{B}$, the matrix model should interpolate between the tube metric for type IIA NS five-branes and the corresponding metric for type IIB NS five-branes.

Let us place all the hypermultiplets at the same point on $x_{2}$. The degrees of freedom relevant for the decoupled physics consist of the hypermultiplets, $Q_{A}^{f}$, the three scalars $\phi_{3}, \phi_{4}, \phi_{5}$ parametrizing motion within the brane, and a scalar parametrizing motion on the compact direction with radius $R_{B}$. The choice of this final scalar depends on the limit we choose for $R_{B}$. Type IIA and IIB NS five-branes wrapped on a longitudinal circle are equivalent by T-duality. Taking the limit $R_{B} \rightarrow 0$ together with the limits in (2.3) should decouple the bulk physics and result in a matrix description of type IIA NS five-branes. Let us check this is the case. In this limit, the radius $\Sigma_{1}$ is fixed but $\Sigma_{2}$ goes to zero and the theory begins to look 1+1-dimensional. As we will check below, in this limit the natural fourth scalar parametrizing motion on $x_{2}$ is the scalar $\phi_{W}$ for the Wilson line around $x_{2}$. In this limit, $\phi_{W}$ combines with $\phi_{3}, \phi_{4}, \phi_{5}$ into a $1+1$-dimensional hypermultiplet. The four scalars frozen at the position of the five-branes combine with the gauge-fields into a $1+1$-dimensional vector multiplet. To determine whether the effective dynamics is really determined by a $1+1$-dimensional model, we need to study the dimensionless quantity

$$
\gamma=g_{Y M}^{2} \Sigma_{2}=\frac{1}{\left(R_{2} M_{s}\right)^{2}},
$$

in a way analogous to [27,[]. As $R_{2} \rightarrow \infty, \gamma \ll 1$ and theory becomes two-dimensional while the effective gauge interactions are weak. In this case, it is reasonable to dimensionally reduce to a $1+1$-dimensional model with $U(N)$ gauge symmetry, an adjoint hypermultiplet, and $k$ fundamental hypermultiplets. We can check that the period for $\phi_{W}$ really decompactifies in this limit. On reduction, the kinetic term for this scalar becomes,

$$
\frac{2 \pi \Sigma_{2}}{g_{Y M}^{2}} \int d t d x_{1}\left(\dot{\phi}_{W}\right)^{2}
$$


where $\phi_{W} \sim \phi_{W}+1 / \Sigma_{2}$. Rescaling the kinetic term to obtain a dimensionless scalar shows that the period for the circle is proportional to $1 / \sqrt{\gamma}$ and so decompactifies as $\gamma \rightarrow 0$. Since the scalars in the vector multiplet are frozen, the dynamics corresponding to the decoupled physics comes from the Higgs branch. The effective $1+1$-dimensional coupling constant,

$$
g_{e f f}^{2}=g_{Y M}^{2} / 2 \pi \Sigma_{2}
$$

also goes to infinity. The theory then flows to the conformal field theory of the Higgs branch in accord with [5],6].

In the opposite limit where $R_{2} \rightarrow 0$, we obtain type IIB five-branes. Again $\Sigma_{1}$ is fixed, while $\Sigma_{2} \rightarrow 0$. The bulk Yang-Mills coupling is again driven to infinity. In this limit where $\gamma \gg 1$, the three-dimensional gauge dynamics cannot be ignored. Even though $\Sigma_{2} \rightarrow 0$, we cannot reduce the model trivially to a $1+1$-dimensional theory. The bulk coupling constant (2.6) is driven to infinity so the theory flows to a particular superconformal field theory for every choice of $N$ and $k>1$.

In this limit, for the abelian case where $N=1$, the natural scalar to use for motion on $x_{B}$ is the dualized gauge-field, $\phi_{D}$, rather than $\phi_{W}$. After rescaling the kinetic term for $\phi_{D}$, we see that the period is proportional to $\sqrt{\gamma}$. On strictly physical grounds, the direction $x_{B}$ must become symmetric with the three non-compact directions of the longitudinal five-brane as $R_{B} \rightarrow \infty$. This implies that in the strong coupling limit, the $\operatorname{Spin}(3)$ flavor symmetry is enhanced to $\operatorname{Spin}(4)$ with $\phi_{D}$ combining with $\phi_{3}, \phi_{4}, \phi_{5}$. A similar enhancement must also occur for the non-abelian case. The use of the dual scalar rather than the scalar for the Wilson line on $x_{2}$ played an important role in matrix formulation of the type IIB string. The chiral spacetime supersymmetries came about because the extra dimension involved the dual scalar. Here the situation is analogous. In the $R_{2} \rightarrow \infty$, we are describing a theory with $(2,0)$ supersymmetry in six dimensions; in the opposite limit, we obtain a theory with $(1,1)$ supersymmmetry. However, there is an important subtlety in this case. The localized interactions treat the two gauge-fields $A_{1}$ and $A_{2}$ differently. The interaction breaks Lorentz invariance so the usual dualization procedure cannot be straightforwardly applied to the hypermultiplet interactions, even in the abelian case. The strong coupling brane picture in terms of $\mathrm{M}$ theory five-branes and two-branes suggests a possible way of obtaining the dualized interaction by starting with the membrane action rather than with the $\mathrm{D}$ 2-brane action.

For the case $N=1$, the $2+1$-dimensional gauge dynamics are unlikely to matter. In this case, all the bulk fields appear quadratically in the Lagrangian. The coupling can then 
be scaled into the $1+1$-dimensional quartic interactions. The decoupled physics should then be governed by a 1+1-dimensional sigma model with a target space metric isomorphic to the metric on the moduli space of one instanton in $S U(k)$ gauge theory. For example, for $k=2$ the target space would be,

$$
\mathbb{R}^{4} \times \mathbb{R}^{4} / \mathbb{Z}_{2}
$$

At first sight, this may seem to coincide with the sigma model for the matrix model for the $(2,0)$ string theory with $N=1$. However, again the $\mathbb{R}^{4}$ contains the dual scalar and so the fermion content should differ. It seems likely that the $\theta$ angle for this orbifold theory is zero since the theory should not describe free strings in six dimensions [6]. It is tempting to conjecture that for higher $N$, the decoupled physics will be described by a sigma model on the moduli space of $\mathrm{N}$ instantons in $S U(k)$ gauge theory. The difference between the $(2,0)$ and $(1,1)$ cases again encoded in the structure of the fermions. It would be interesting to explore this possibility further.

\section{The Type IIA Matrix String on an ADE Singularity}

To determine the dual realization of the superconformal field theory describing the decoupled physics, let us study case two. Some related comments have appeared in [28,29]. In this description, we have NS five-branes in type IIB with a compact transverse circle. On T-duality, the system becomes a configuration of Kaluza-Klein monopoles in type IIA string theory [30]. The monopole solution is constructed by taking the spacetime metric to be a product of a flat metric for five-dimensions with a multi-Taub-NUT metric [31]. We will briefly recall the main features of the multi-Taub-NUT metric which have appeared recently in various discussions of Kaluza-Klein monopoles in string theory and matrix theory [32, 34,29, [7]. The metric is determined by a single function $V$,

$$
d s^{2}=V(x) d \vec{x}^{2}+V(x)^{-1}(d \theta+\vec{A} \cdot d \vec{x})^{2},
$$

where $\nabla V=\nabla \times \vec{A}$. The function $V$ can be written

$$
V=1+r \sum_{i=1}^{k} \frac{1}{\left|\vec{x}-\vec{x}^{i}\right|} .
$$

The free parameter $r$ sets the scale for this hyperKähler metric, and the coordinate $\theta$ has a period proportional to $r$. The $k$ branes are located at the points $\vec{x}^{i}$. The parameter 
$r=R_{2}$ in this case. When we have $k$ coincident branes, the multi-Taub-Nut has an $A_{k-1}$ singularity at the location of the coincident branes. Key to this story is the independence of the decoupled physics from the value of $R_{2}$ [7]. We are free to take the limit $R_{2} \rightarrow \infty$ which decompactifies the circle coordinatized by $\theta$ everywhere except at the location of the branes. The space then becomes $\mathbb{R}^{4} / \mathbb{Z}_{k}$.

This is fortunate since we do not currently know how to provide a matrix model description for Kaluza-Klein monopoles in type IIA string theory. Such a matrix description should be obtained by compactifying a transverse direction, say $x^{9}$, to the type IIB NS five-brane. The corresponding matrix model is $3+1$-dimensional with $2+1$-dimensional hypermultiplets. This theory should be well-defined since we have compactified only two transverse directions to the longitudinal five-brane. This is to be contrasted with the matrix model for an $\mathrm{M}$ theory five-brane with three compact transverse dimensions. The matrix model is similar but the hypermultiplets are not localized. The gauge theory is then not asymptotically free and needs definition in the ultra-violet. This problem can also be associated to the deficit angle generated by seven-branes by T-dualizing the $k$ four-branes on a transverse $T^{3}$. It is natural to attempt to define this matrix theory in terms of the six-dimensional theory corresponding to $N \operatorname{Spin}(32)$ instantons compactified on $T^{2}$, which at low energies has $S p(N)$ gauge symmetry. This is the decoupled theory living on parallel type I five-branes [3]. This definition only makes sense for sixteen or fewer five-branes, and also leads us down the road of 'matrix models for matrix models.' Fortunately, when the hypermultiplets are localized, we expect no such problems.

For the decoupled physics, we actually only need a matrix description of the type IIA string on an $A_{k-1}$ singularity which has been supplied in 35,28$]$. The matrix theory is a $1+1$-dimensional gauge theory with $(4,4)$ supersymmetry and gauge group,

$$
U\left(N_{i_{1}}\right) \times \cdots \times U\left(N_{i_{k}}\right) .
$$

Each factor is associated to a node of the extended Dynkin diagram; see, for example [11,35]. For the $A_{k-1}$ case, $N_{i_{1}}=\ldots=N_{i_{k}}=N$. The matter content can also be read from the extended Dynkin diagram where we obtain a hypermultiplet for each link in the diagram. This can be summarized in an adjacency matrix whose elements, $a_{i j}$, are one

2 A slight modification of this original conjecture has recently been proposed in [8], but our results seem to be compatible with the gauge groups and matter content originally suggested in [35]. I wish to thank K. Intriligator for discussions on this point. 
when there is a link between the $i^{\text {th }}$ and $j^{t h}$ node and zero otherwise. The hypermultiplets appear in the representations, $\oplus_{i j} a_{i j}\left(N_{i}, \bar{N}_{j}\right)$. The $k$ hypermultiplets are chosen so that the Higgs branch corresponds to a product of $A_{k-1}$ singularities. The singularities can be resolved by turning on Fayet-Iliopoulos parameters. Our interest, however, is not with the Higgs branch which describes type IIA strings moving on a space-time which is a product of $\mathbb{R}^{4}$ with an ALE space. When the Fayet-Iliopoulos parameters are set to zero so the space is singular, a new branch should appear in the matrix description. Further, the conformal field theory for this branch should decouple from the spacetime physics. The Coulomb branch of the current matrix proposal for the type IIA string on an $A_{k-1}$ singularity satisfies these requirements. It disappears when the singularity is resolved, and it decouples from the Higgs branch in the infra-red. There are two distinct infra-red limits because the symmetry flowing to the $\mathrm{R}$ charge of the $(4,4)$ superconformal algebra is necessarily different for the Higgs and Coulomb branches [1]. On the Coulomb branch, the strings are trapped at the location of the singularity. That is precisely the picture we desire for the decoupled sector.

The Coulomb branch is a $4 N k$-dimensional space with a metric that has a tube-like structure. For $N=1$ and $k=2$, the Coulomb branch is one-dimensional and the metric can be determined essentially by symmetries [27]. One $U(1)$ vector multiplet decouples, leaving a $U(1)$ gauge theory with two electrons. The matrix theory is then a $1+1$-dimensional theory on a circle of radius $\Sigma_{1}$ given in (2.7). In the decoupling limit, the coupling constant for the gauge theory is driven to infinity. The target space is a product of $\mathbb{R}^{4}$ with a fourdimensional space with metric proportional to $\frac{1}{r^{2}}$ and non-trivial torsion [27. We might ask whether it is actually valid to restrict to the description in terms of a metric on moduli space for small $r$, since that is roughly a Born-Oppenheimer approximation. A similar argument in the dimensional reduction of this gauge system to quantum mechanics would seem to yield a singular $\frac{1}{r^{3}}$ metric fixed uniquely by a global $\operatorname{Spin}(5)$ symmetry [36]. However, there we know that the description of the physics in terms of a metric on moduli space breaks down. There is actually a normalizable ground state wave-function, and the physics is quite non-singular [37]. There is clearly much to be understood about these singular conformal field theories.

Now it has been conjectured in [1,27 and argued in [38 that there should be a dual realization in which the tube is absent. That dual realization was presented in the previous section, and argued to be a sigma model on $\mathbb{R}^{4} \times \mathbb{R}^{4} / \mathbb{Z}_{2}$. Note that there was no torsion in that realization. The subtlety with the tube metric manifests itself in the dual description 
in the guise of a hard to analyze $\theta=0$ orbifold theory. It is important to stress that the duality that we are claiming follows from a legitimate string T-duality: the T-duality taking an NS five-brane with a compact transverse circle to a Kaluza-Klein monopole. This dual realization of superconformal field theories seems to be the natural analogue of the three-dimensional mirror symmetry found by Intriligator and Seiberg [11]. It equates a $1+1$-dimensional limit of the 'Higgs' branch for a $2+1$-dimensional theory with localized hypermultiplets with the Coulomb branch conformal field theory of a $(4,4)$ gauge theory. This duality extends to the family of superconformal field theories parametrized by $N$ and $k>1$, and generally exchanges a metric with torsion for a hyperKähler metric. This duality is clearly related to conventional two-dimensional mirror symmetry [39], where a theory with $(2,2)$ supersymmetry can often be realized in terms of sigma models on two or more distinct target spaces. However, in those cases, the target space is typically compact.

Lastly, we can define the decoupled $(1,1)$ string theory corresponding to the $D$ and $E$ singularities by using the Coulomb branch of the type IIA matrix string propagating on those singular spaces. The gauge groups and matter content have been described in [11,40]. The hypermultiplets are chosen so that the Higgs branch is again a product of $D$ or $E$ singularities. Again the corresponding Coulomb branch conformal field theory will describe the decoupled physics. For the $D_{k}$ case, a dual description of the Coulomb branch conformal field theory naturally follows by considering NS five-branes and orientifold planes. The $E_{6}, E_{7}, E_{8}$ theories are likely to have more exotic dual realizations.

\section{Acknowledgements}

It is my pleasure to thank J. Brodie, K. Intriligator, D. Morrison, R. Plesser and particularly O. Ganor, N. Seiberg and E. Witten for helpful discussions. This work is supported by NSF grant DMS-9627351. 


\section{References}

[1] E. Witten, "Some Comments on String Dynamics," hep-th/9507121.

[2] A. Strominger, "Open P-Branes," hep-th/9512059, Phys. Lett. B383 (1996) 44.

[3] N. Seiberg, "New Theories in Six Dimensions and Matrix Description of M-theory on $T^{5}$ and $T^{5} / \mathbb{Z}_{2}, "$ hep-th/9705221.

[4] N. Seiberg, "Notes on Theories with 16 Supercharges," hep-th/9705117.

[5] O. Aharony, M. Berkooz, S. Kachru, N. Seiberg, and E. Silverstein, "Matrix Description of Interacting Theories in Six Dimensions," hep-th/9707079.

[6] E. Witten, "On The Conformal Field Theory of The Higgs Branch," hep-th/9707093.

[7] N. Seiberg and S. Sethi, "Comments on Neveu-Schwarz Five-Branes," hep-th/9708085.

[8] K. Intriligator, "New String Theories in Six Dimensions via Branes at Orbifold Singularities," hep-th/9708117.

[9] D. Lowe, " $E_{8} \times E_{8}$ Small Instantons in Matrix Theory," hep-th/9709015;

O. Aharony, M. Berkooz, S. Kachru and E. Silverstein, "Matrix Description of $(1,0)$ Theories in Six Dimensions," hep-th/9709118.

[10] R. Dijkgraaf, E. Verlinde and H. Verlinde, "BPS Spectrum of the Five-Brane and Black Hole Entropy," hep-th/9603126, Nucl. Phys. B486 (1997) 77; "BPS Quantization of the Five-Brane," hep-th/9604055, Nucl. Phys. B486 (1997) 89; "5D Black Holes and Matrix Strings," hep-th/9704018.

[11] K. Intriligator and N. Seiberg, "Mirror Symmetry in Three Dimensional Gauge Theories," hep-th/9607207, Phys. Lett. B386 (1996) 513.

[12] E. Witten, to appear.

[13] J. Brodie, "Two Dimensional Mirror Symmetry from M-theory," hep-th/9709228.

[14] P. Aspinwall, "Some Relationships Between Dualities in String Theory," hep-th/9508154, Nucl. Phys. Proc. Suppl. 46 (1996) 30;

J. Schwarz, "The Power of M Theory," hep-th/9510086, Phys. Lett. B367 (1996) 97.

[15] O. Ganor and S. Sethi, to appear.

[16] S. Sethi and L. Susskind, "Rotational Invariance in the M(atrix) Formulation of Type IIB Theory," hep-th/9702101, Phys. Lett. B400 (1997) 265.

[17] T. Banks and N. Seiberg, "Strings from Matrices," hep-th/9702187, Nucl. Phys. B497 (1997) 41.

[18] T. Banks, W. Fischler, S. H. Shenker, and L. Susskind, "M Theory As A Matrix Model: A Conjecture," hep-th/9610043, Phys. Rev. D55 (1997) 5112.

[19] L. Susskind, "Another Conjecture about M(atrix) Theory," hep-th/9704080.

[20] M. Berkooz and M. Douglas, "Five-branes in M(atrix) Theory," hep-th/9610236, Phys. Lett. B395 (1997) 196.

[21] W. Taylor IV, "D-Brane Field Theory on Compact Space," hep-th/9611042, Phys. Lett. B394 (1997) 283. 
[22] C.G. Callan, J.A. Harvey, A. Strominger, "Supersymmetric String Solitons," hepth/9112030, Nucl. Phys. B359 (1991) 611;

S.-J. Rey, in "The Proc. of the Tuscaloosa Workshop 1989," 291; Phys. Rev. D43 (1991) 526; S.-J. Rey, In DPF Conf. 1991, 876.

[23] J. de Boer, K. Hori, H. Ooguri and Y. Oz, "Mirror Symmetry in Three-Dimensional Gauge Theories, Quivers and D-branes," hep-th/9611063, Nucl. Phys. B493 (1997) 101.

[24] E. Witten, "Solutions Of Four-Dimensional Field Theories Via M Theory, " hepth/9703166.

[25] O. Ganor, S. Ramgoolam and W. Taylor IV, "Branes, Fluxes and Duality in M(atrix)Theory," hep-th/9611202, Nucl. Phys. B492 (1997) 191.

[26] A. Kapustin and S. Sethi, to appear.

[27] D.-E. Diaconescu and N. Seiberg, "The Coulomb Branch of $(4,4)$ Supersymmetric Field Theories in Two Dimensions," hep-th/9707158.

[28] M. Douglas, "Enhanced Gauge Symmetry in M(atrix) Theory," hep-th/9612126;

W. Fischler and A. Rajaraman, "M(atrix) String Theory on K3," hep-th/9704123.

[29] A. Hanany and G. Lifschytz, "M(atrix) Theory on $T^{6}$ and a m(atrix) Theory Description of KK Monopoles," hep-th/9708037.

[30] T. Banks, M. Dine, H. Dykstra and W. Fischler, "Magnetic Monopole Solutions of String Theory," Phys. Lett. B212 (1988) 45;

C. Hull and P. Townsend, "Unity of Superstring Dualities," hep-th/9410167, Nucl. Phys. B438 (109) 1995.

[31] S. Hawking, "Gravitational Instantons," Phys. Lett. 60A (1977) 81;

G. Gibbons and S. Hawking, "Classification of Gravitational Instanton Symmetries," Comm. Math. Phys. 66 (1979) 291;

R. Sorkin, "Kaluza-Klein Monopole," Phys. Rev. Lett.51 (1983) 87;

D. Gross and M. Perry, "Magnetic Monopoles in Kaluza-Klein Theories," Nucl. Phys. B226 (1983) 29.

[32] A. Sen, "Kaluza-Klein Dyons in String Theory," hep-th/9705212; "A Note on Enhanced Gauge Symmetries in M and String Theory," hep-th/9707123; "Dynamics of Multiple Kaluza-Klein Monopoles in M and String Theory," hep-th/9707042.

[33] C. Hull, "Gravitational Duality, Branes and Charges," hep-th/9705162;

E. Bergshoeff, B. Janssen, and T. Ortin, "Kaluza-Klein Monopoles and Gauged Sigma Models," hep-th/9706117;

Y. Imamura, "Born-Infeld Action and Chern-Simons Term from Kaluza-Klein Monopole in M-theory," hep-th/9706144;

R. Gregory, J. Harvey and G. Moore, "Unwinding strings and T-duality of KaluzaKlein and H-Monopoles," hep-th/9708086. 
[34] D. Berenstein, R. Corrado and J. Distler, "On the Moduli Spaces of M(atrix)-Theory Compactifications," hep-th/9704087;

S. Elitzur, A. Giveon, D. Kutasov and E. Rabinovici, "Algebraic Aspects of Matrix Theory on $T^{d}$," hep-th/9707217;

A. Losev, G. Moore, and S. Shatashvili, "M \& m's ," hep-th/9707250;

I. Brunner and A. Karch, "Matrix Description of M-theory on $T^{6}$," hep-th/9707259.

[35] M. Douglas and G. Moore, "D-Branes, Quivers, and ALE Instantons," hep-th/9603167.

[36] D.-E. Diaconescu and R. Entin, "A Non-Renormalization Theorem for the d=1, N=8 Vector Multiplet," hep-th/9706059.

[37] S. Sethi and M. Stern, "A Comment on the Spectrum of H-Monopoles," hepth/9607145, Phys. Lett. B398 (1997) 47.

[38] H. Ooguri, C. Vafa, "Two Dimensional Black Hole and Singularities of Calabi-Yau Manifolds," Nucl.Phys. B463 (1996) 55, hep-th/9511164;

D. Kutasov, "Orbifolds and Solitons," Phys. Lett B383 (1996) 48, hep-th/9512145.

[39] B. Greene and R. Plesser, "Duality in Calabi-Yau Moduli Space," Nucl. Phys. B338 (1990) 15;

W. Lerche, C. Vafa and N. Warner, "Chiral Rings in N=2 Superconformal Theories," Nucl. Phys. B324 (1989) 427.

[40] C. Johnson and R. Myers, "Aspects of Type IIB Theory on ALE Spaces," hepth/9610140, Phys. Rev. D55 (1997) 6382. 